\title{
G-protein $\beta 2$ subunit interacts with mitofusin 1 to regulate mitochondrial fusion
}

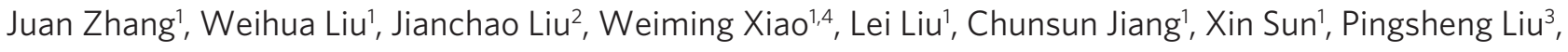 \\ Yushan Zhu',2, Chuanmao Zhang ${ }^{4} \&$ Quan Chen ${ }^{1,2}$
}

Mitofusins (Mfns) mediate the fusion of mitochondrial membranes. However, little is known about how Mfns are regulated to control mitochondrial fusion, which is a multistep process requiring tethering and docking of the outer membranes of two mitochondria. In this study, we report that guanine nucleotide binding protein- $\beta$ subunit 2 (GB2), a WD40 repeats protein and a member of the $\beta$-subunits of the heterotrimeric $G$ proteins, has a crucial function in mitochondrial fusion. $G \beta 2$ was found to be enriched on the surface of mitochondria and interacted with mitofusin 1 (Mfn1) specifically. $\mathrm{G} \beta 2$ also regulated the mobility of Mfn1 on the surface of the mitochondrial membrane and affected the mitochondrial fusion. Depletion of endogenous $\mathrm{G} \beta 2$ resulted in mitochondrial fragmentation, which could be rescued by exogenous $\mathrm{G} \beta 2$. These findings have thus uncovered a novel role of $\mathrm{G} \beta 2$ in regulating mitochondrial fusion through its interaction with Mfn1.

\footnotetext{
1 The Joint Laboratory of Apoptosis and Cancer Biology, The State Key Laboratory of Biomembrane and Membrane Biotechnology, Chinese Academy of Sciences, Beijing 100101, China. ${ }^{2}$ College of Life Sciences, Nankai University, Tianjin 300071, China. ${ }^{3}$ Institute of Biophysics, Chinese Academy of Sciences, Beijing 100101, China. ${ }^{4}$ The State Key Laboratory of Biomembrane and Membrane Biotechnology, Peking University, Beijing 100871, China. Correspondence and requests for materials should be addressed to Q.C. (email: chenq@ioz.ac.cn).
} 
M itochondria are dynamic organelles undergoing constant fusion and fission, and dysregulation of mitochondrial dynamics has been linked to altered mitochondrial physiology, abnormal mitochondrial DNA maintenance, apoptosis ${ }^{1,2}$ and neuromuscular diseases ${ }^{3,4}$. These two opposing processes are regulated by two members of the dynamin family: mitofusins (Mfns) and dynamin-related guanosine triphosphatases (GTPases) (Drp1) $)^{5,6}$. Proper control of mitochondrial fission and fusion is vital to both the integrity of the mitochondrial morphology and their functions ${ }^{7-11}$. Several WD40 proteins, such as Mdv1 and Caf4, are found to interact with Dnm1, a major mediator for mitochondrial fission in yeast, to determine the localization of Dnm1 on the mitochondrial surface ${ }^{12}$ and modulate the process of fission, at least in the yeast system ${ }^{13}$. Mfn 1/2, two mitochondrial GTPases in the dynamin family, mediate the fusion of mitochondrial membrane. During these processes, Mfn1 and Mfn2 act in a trans-manner leading to a closer apposition of the membranes for completing the fusion ${ }^{14}$. Removal of the GTPase domain of Mfn1 is still able to induce the aggregation of mitochondria without complete fusion ${ }^{14,15}$, suggesting that domains other than GTPase activity are required for mitochondrial docking or tethering. We reasoned that there may be other factors that act to coordinate Mfns for mitochondrial fusion.

In this study, we report that guanine nucleotide binding protein- $\beta$ subunit 2 (G $\beta 2$ ), a member of the $\beta$-subunits of the heterotrimeric $\mathrm{G}$ proteins and a WD40 protein, has a crucial function for mitochondrial fusion. G $\beta 2$ is partially found on the surface of mitochondria and physically interacts with Mfn1. Depletion of G $\beta 2$ perturbs mitochondrial morphology and decrease mitochondrial fusion rate. $\mathrm{G} \beta 2$ acts by limiting the membrane mobility of Mfn1 at the mitochondrial surface to regulate the process of mitochondrial fusion. These findings demonstrated that G $\beta 2$ interacts with $\mathrm{mfn} 1$ to regulate mitochondrial fusion.

\section{Results}

G及2 localizes to the surface of mitochondria. To identify potential proteins regulating Mfns, we used a yeast two hybrid assay to identify potential molecules, which may interact with Mfn 1 or 2 by using the full-length Mfn1 and Mfn 2, or different domains of Mfn1 as baits. As a result, we obtained 91 positive clones, 3 of which encoded G $\beta 2$ when the fragment of aa241-387 was used as a bait. $\mathrm{G} \beta 2$ interaction with Mfn1 is specific, as reconstitution of $\mathrm{AD}$ (active domain)-G $\beta 2$ (in the fish vector) with $\mathrm{BD}$ (binding domain)-Mfn1 (in the bait vector), but not with BD-Mfn2, gave a strong positive result (see Supplementary Fig. S1a).

Structural analysis has revealed that the G $\beta$ subunits are WD40 repeat proteins with a toroidal structure containing seven-bladed $\beta$-propellers ${ }^{16}$. The unique toriodal $\beta$-propeller structure defines surfaces for interactions with numerous receptors, $G \alpha$ and many other effectors ${ }^{17}$. Despite accumulating evidence that has shown $\mathrm{G} \beta \gamma$ dimers exert its functions on various effectors as the G $\alpha$ proteins $\mathrm{do}^{18-20}$, the role of $\mathrm{G} \beta 2$ in mitochondrial dynamics is largely unknown. Thus, we first examined if G $\beta 2$ and Mfns are co-localized in mitochondria by immunostaining. Indeed, our data revealed that endogenous G 32 was localized in mitochondria in HeLa (Fig. 1a) and SH-SY5Y neuroblastoma cells (Supplementary Fig. S1b). In contrast, a specific antibody to $G \beta 1$, most similar to $G \beta 2$ in amino acids identity among all G $\beta$ subunits, showed diffused staining in HeLa cells (Fig. 1b). Confocal microscopic analysis showed that G $\beta 2$ appeared to have a patchy distribution along the mitochondrial tubular structure, and this observation was substantiated by threedimensional analysis using the scanned confocal images (Fig. 1a). To further ascertain that G $\beta 2$ is indeed localized in mitochondria, we performed a gradient fractionation assay. HeLa cells were homogenized with a Dounce homogenizer and the cell lysates were separated into the crude mitochondrial fraction, $10,000 \times g$ supernatant $\left(\mathrm{C}_{2}\right)$ containing plasma membrane, the microsomal fraction, as well as cytosolic proteins. The crude mitochondrial fraction and $\mathrm{C}_{2}$ were further fractionated by Percoll density gradient centrifugation to obtain pure mitochondrial fraction $(\mathrm{M})$, plasma membrane fraction $(\mathrm{P})$ and the cytosol $\left(\mathrm{C}_{1}\right)$. The purity of the mitochondrial fractions was tested by loading equal amount of proteins onto a gel validated by using mitochondrial-specific markers such as VDAC1 and Mfn1, and by specific markers for endoplasmic reticulum (p62 (ref. 21)), Golgi (GM130 (ref. 22)). As shown in Figure 1c, Gß2, but not $G \beta 1$, was significantly enriched in the purified mitochondrial fraction compared with other fractions (enrichment 48.5\%). G $\gamma 2$ was also enriched in the purified mitochondrial fraction, whereas G $\gamma 1$

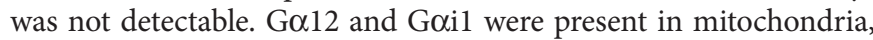
which were consistent with previous reports ${ }^{23,24}$, and other membrane fractions. To determine the precise localization of G $\beta 2$ on the outer membrane, we incubated intact mitochondria isolated from HeLa cells with protease K. Our data showed that G $\beta 2$, similar to Tom20 and Mfn1 that are mitochondrial outer membrane proteins, was sensitive to protease $\mathrm{K}$ (Fig. 1d). Furthermore, G $\beta 2$ was resistant to $\mathrm{NaHCO}_{3}$ treatment, suggesting that $\mathrm{G} \beta 2$ does not adhere to the outer membrane of the mitochondria nonspecifically ${ }^{25}$. Semiquantitative PCR analysis showed that G $\beta 2$ is expressed at high levels in both HeLa and SH-SY5Y cells (Fig. 1e-g, Supplementary Fig. S1c-e).

G及2 physically interacts with Mfn 1 . We next determined whether there is indeed a physical interaction between G 32 and Mfn1. Endogenous G $\beta 2$ was coimmunoprecipitated with Mfn1 but not with normal mouse immunoglobulin-G from HeLa cell lysate. Importantly, depletion of $\mathrm{G} \beta 2$ in the cells by short hairpin RNA (shRNA) abolished the interaction, whereas GB2 could be coimmunoprecipitated with Mfn1 after G 32 was reintroduced into the shRNA cells, and vice versa, when Mfn 1 was used as the bait (Fig. 2a,b). However, we failed to detect an interaction between

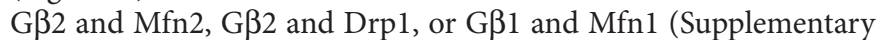
Fig. S2a-c) or between $G \beta 2$ and G $\alpha 12$, which is reportedly localized in mitochondria ${ }^{24}$. There was a weak interaction of G $\beta 2$ with Goil, although the significance of this interaction remains to be determined. To validate our findings that $\mathrm{G} \beta 2$ interacted directly with Mfn1, we performed two independent fluorescence resonance energy transfer (FRET) analyses on HeLa cells co-transfected with Mfn1-CFP and Gß2-YFP. In the cells, the expressed Mfn1-CFP was specifically localized in mitochondria, whereas a majority of YFP-Gß2 was found in mitochondria (Supplementary Fig. S2d). In this assay, the fluorescent intensity of the donor CFP was measured before and after the fluorescence of the acceptor YFP was photobleached (quenched). As shown in Figure 2c, the fluorescent intensity of CFP was remarkably increased after the fluorescence of YFP was completely quenched by a laser beam. This observation suggested that there was a direct interaction between Mfn1-CFP and G $\beta 2$-YFP in the cells. The energy transfer efficiency from CFP to YFP obtained by using the equation described ${ }^{26}$ was $33.9 \%$. In contrast, energy transfer efficiency from Mfn1-CFP to Gß1-YFP was significantly lower (only $\sim 10 \%$ ) (Fig. 2d). Taken together, our data demonstrate that Mfn1 and G $\beta 2$ have a physical interaction and that the interaction is specific. We next determined the structural domains responsible for the interaction of G 32 and Mfn1. Co-immunoprecipitation of truncated mutants of Mfn1 and Flagtagged G 32 in HeLa cells revealed that, consistent with the yeast two hybrid assay, the domain of Mfn 1 from aa241 to 350 was necessary for the interaction between Mfn1 and G $\beta 2$ (Fig. 3a,b). Also, the Mfn1 mutant lacking the interaction domain had diminished FRET (Supplementary Fig. S2e). Importantly, we found that Mfn1 lacking the interacting domain from aa241-350 failed to induce mitochondrial aggregation compared with that of wild-type Mfn1 (Fig. 3c), whereas GTPase dead mutants Mfn1 T109A and K88T were able to induce mitochondrial aggregation (Supplementary Fig. S2f). Our results thus identify that, in addition to the heptad repeat region 
a

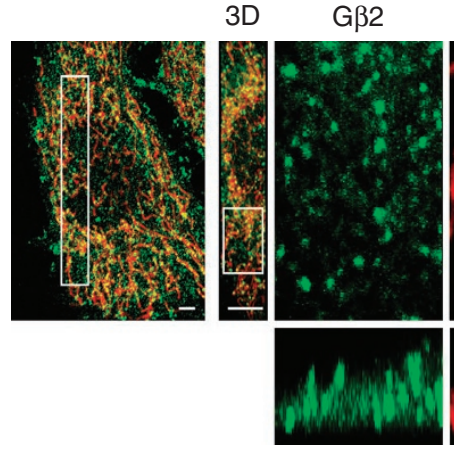

C

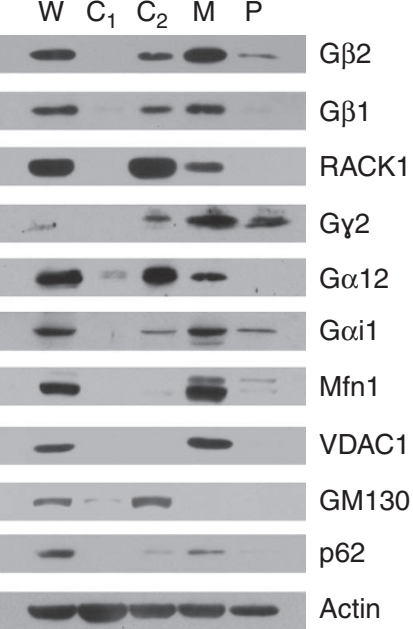

MitoTracker

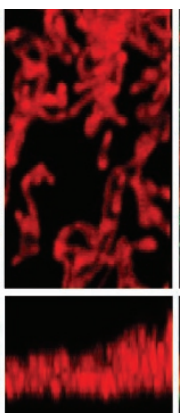

d
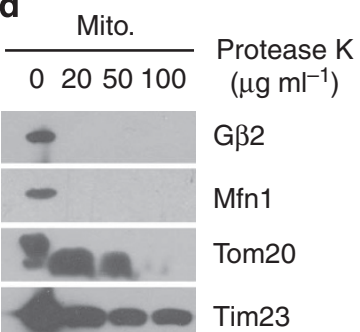

Gß2

Mfn1

Tom20

Tim23
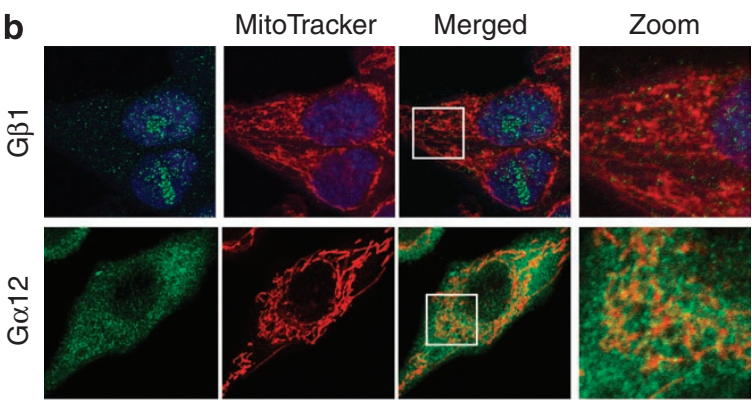

$\underset{\mathbb{1}}{\frac{1}{1}}$
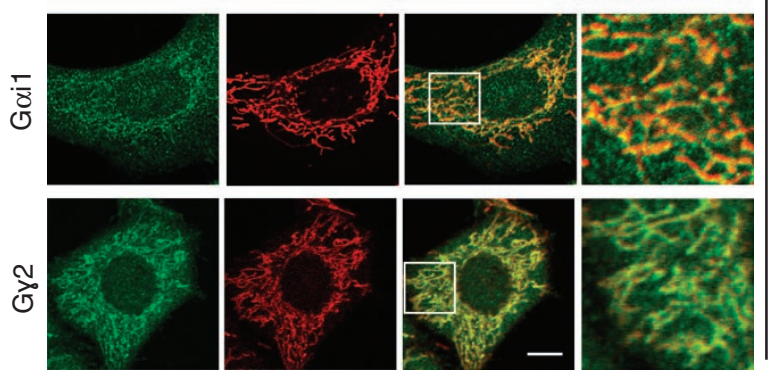
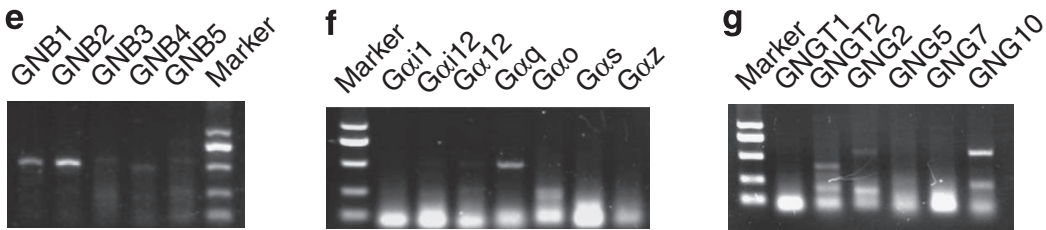

Figure 1 | Distribution of $\mathbf{G} \mathbf{2} 2$ protein on the surface of mitochondria. (a) Immunostaining with a specific antibody shows the localization of G $\beta 2$ in HeLa cells. Cells were stained with $20 \mathrm{nM}$ Mitotracker Red, and then immuno-labelled with anti-G $\beta 2$ (green) and visualized with a two-photon microscope (Zeiss, LSM 510). The boxed area on the upper left was scanned along the $Z$ axis, and the images were superposed to form the three-dimensional (3D) image. The boxed area in the 3D image is shown at higher magnification in the upper right panels. The lower right shows images of $Y$ to $Z$ dimension. Scale bar,

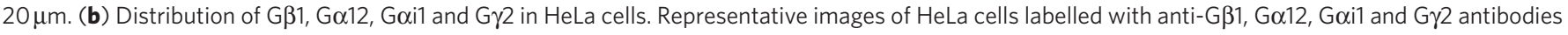
(green) and stained with $20 \mathrm{nM}$ Mitotracker Red. Scale bar, $10 \mu \mathrm{m}$. (c) Distribution of $\mathrm{G} \beta 2$ and other $\mathrm{G}$ proteins in the mitochondrial fraction. Equal amounts of the whole cell lysate $(W)$, cytosol $\left(C_{1}\right)$, light membrane $\left(C_{2}\right)$, mitochondria $(M)$ and plasma membrane $(P)$ fractions were loaded and analysed for the distribution of $\mathrm{G} \beta 2$, $\mathrm{G} \gamma 2$ and other $\mathrm{G}$ proteins by western blot using specific antibodies. The purity of isolated mitochondria was determined by using the markers for distinct organelles: Mfn1, VDAC1 (mitochondria), GM130 (Golgi), p62 (endoplasmic reticulum) and actin (cytoplasm). (d) Protease K assay for G $\beta 2$ 's localization on mitochondria. Mitochondrial fractionation obtained from HeLa cells was subjected to Protease K digestion for 30 min with different concentrations as indicated. G 2 and other mitochondrial proteins such as Mfn1, Tom20 (outer mitochondrial membrane, OMM) and Tim23 (inner mitochondrial membrane, IMM) were analysed by western blot with corresponding antibodies. Equal amounts of protein were loaded for Protease $\mathrm{K}$ treatment. (e) $G \beta 2$ is the most abundant subunit of $G \beta$ proteins in HeLa cells. mRNA levels of distinct $G \beta$ subunits were analysed by semiquantitative PCR in HeLa cells. $(\mathbf{f}, \mathbf{g})$ The mRNA levels of $\mathrm{G} \alpha, \mathrm{G} \gamma$ subunits in the cells were analysed by semiquantitative PCR (right panel). The size markers are 1,000, 750, 500,200 and $100 \mathrm{bp}$, respectively, from top to bottom. All the experiments were repeated at least three times.

(HR2) domain of Mfn $1^{15}$, the domain between the GTPase domain and the HR1 domain is also important for mitochondrial fusion by its interaction with G $\beta 2$. On the other hand, we generated a series of Flag-tagged G $\beta 2$ with various truncations of WD repeats and transfected these constructs into HeLa cells (Fig. 3d), and found that they failed to induce mitochondrial aggregation (Fig. 3e). Co-immunoprecipitation revealed that the deletion of four WD40 repeats of G $\beta 2$ completely abolished its interaction with Mfn1 (Fig. 3f), suggesting that the toroidal structure needs to be intact for their interaction and subsequent mitochondrial fusion.

G $\beta 2$ requires Mfn1 for its effect on mitochondrial dynamics. We then addressed the biological significance of the interaction between G 32 and Mfn1. As shown in Figure 4a, G $\beta 2$ induced mitochondrial aggregation in both wild-type and Mfn2 $2^{-/-}$MEF cells, in which Mfn1 is expressed. However, ectopic expression of G $\beta 2$ failed to induce mitochondrial aggregation in $\mathrm{Mfn}^{-1-}$ cells $\left(\mathrm{Mfn} 1^{-1-}\right.$ or Mfn1\&2 $2^{-/-}$MEF cells). These data support our notion that G $\beta 2$ indeed requires Mfn1 for regulating mitochondrial fusion. A previous study has shown ${ }^{27}$ that mitochondria are spheres or ovals and maintain a certain degree of fusion in $\mathrm{Mfn} 2^{-1-} \mathrm{MEF}$ cells compared with that of $\mathrm{Mfn}^{-1-} \mathrm{MEF}$ cells. Interestingly, G 32 shRNA could further fragment mitochondria, resulting in the size of mitochondria similar to that in $\mathrm{Mfn}^{-/-}$MEFs. In contrast, it had little effect on the mitochondrial morphology in Mfn $1^{-1-}$ MEF cells (Fig. 4b,c). It is also interesting to note that G $\beta 2$ localizes at the specific submitochondrial locations where mitochondria appear to have the tendency of branching along mitochondrial tubular structure (see Fig. 1a). Collectively, these data suggest that G $\beta 2$ regulates the mitochondrial fusion, which requires Mfn1.

G $\beta 2$ regulates the mobility of $\mathrm{Mfn} 1$ on the mitochondria. It is possible that $G \beta 2$ regulates mitochondrial fusion by affecting the distribution of Mfn1 on the microregion of mitochondrial surface and thereby the membrane mobility of Mfn1. To test this hypothesis, we measured the Mfn1 membrane mobility in the 
a
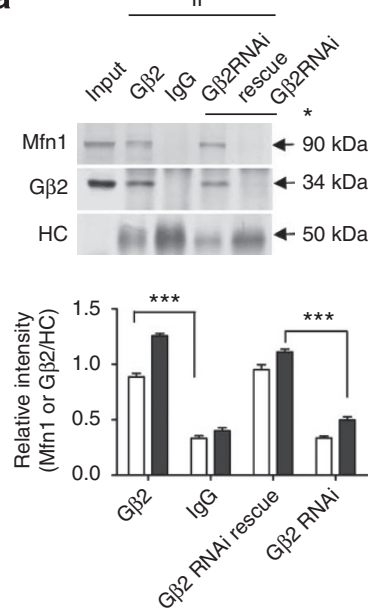

c

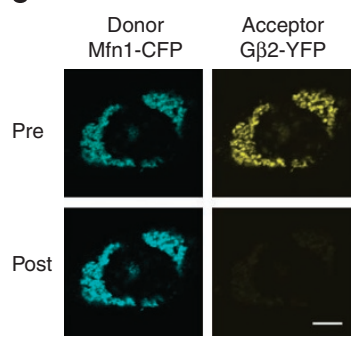

b
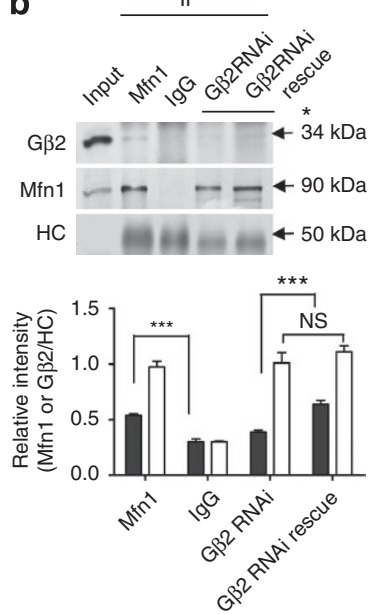

d

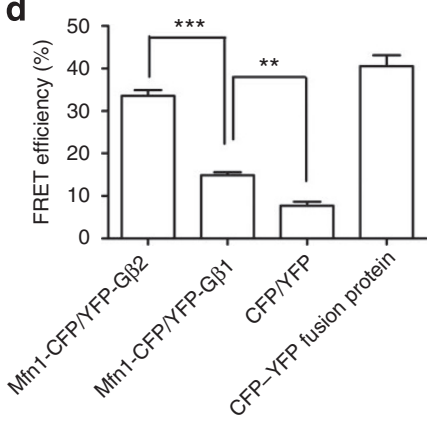

Figure 2 | G $\beta 2$ physically interacts with $\mathbf{M f n} 1$ to induce mitochondrial aggregation. (a) Immunoprecipitation (IP) of $G \beta 2$ with Mfn1. Lysates either from HeLa cells, or cells expressing a shRNA specific to $G \beta 2$ or the latter reintroduced with a mutant of Flag-G $\beta 2$ carrying a mutation for eliminating the RNAi target sequence (the right two lanes marked as asterisk), were incubated with a G $\beta 2$ antibody overnight, followed by western blot with an anti-Mfn1 antibody. The crude lysates from these cells were used as input. (b) Mfn1 coimmunoprecipitates with $\mathrm{G} \beta 2$. The cell lysates were incubated with Mfn1 antibody overnight, followed by western blot with an anti-G $\beta 2$ antibody. Mfn1, G $\beta 2$ and heavy chain $(\mathrm{HC}$ ) levels were quantified by densitomery. Relative intensity were obtained as intensity of bands of Mfn1 (opened box) or G $\beta 2$ (filled box) divided by intensity of heavy chain of relative lines. Error bars represent the s.e.m., $n=3$; ${ }^{\star \star \star}$ for $P<0.0005$, twotailed test. (c) Images of fluorescence intensity from the cells co-transfected with CFP-Mfn1 and YFP-GB2 in CFP and YFP channels before and after photobleaching of YFP. The increase of the CFP fluorescence and dramatic decrease of the YFP fluorescence were observed after the photobleaching, Scale bar, $10 \mu \mathrm{m}$. (d) The histogram shows the FRET efficiency from CFP to YFP in the cells transfected with both CFP-Mfn1 and YFP-G $\beta 2$, CFPMfn1 and YFP-G $\beta 1, C F P-Y F P$ fusion protein and both of CFP and YFP, respectively. Error bars represent the s.e.m., $n=18$; Differences in the mean FRET efficiency between CFP-Mfn1 and YFP-G $\beta 2$, CFP-Mfn1 and YFP-G $\beta 1$, or CFP and YFP were statistically significant at ${ }^{\star \star \star} P<0.0005$, ${ }^{\star \star} P<0.005$, two-tailed $t$-test.

presence or absence of G $\beta 2$ using a FRAP assay. This technique provides a semiquantitative measurement of the movement of the mitochondrial proteins by assessing the depth of bleaching and rate of redistribution of mitochondrial outer membrane-localized yellow/green fluorescence protein (Mfn1-YFP was used in the assay (Supplementary Fig. S3a)) into regions of irreversible fluorophore photobleach ${ }^{28}$. Intriguingly, downregulation of G $\beta 2$ by shRNA in HeLa cells caused an increase of Mfn1 mobility, whereas overexpression of G $\beta 2$ caused a significant decrease in the mobility of Mfn1 (Fig. 4d,e). In addition, G $\beta 2$ shRNA failed

to increase the mobility of Mfn 1 mutants $(\Delta 241-350$ and $\Delta 1-387)$ that lack the interacting domain (Fig. 4f). This finding further supports our notion that their interaction is critical for the mobility of Mfn1 on the outer membrane surface of mitochondria. In contrast, the mobility of Mfn2 showed little change when G $\beta 2$ was downregulated in HeLa cells (Supplementary Fig. S3b,c). Moreover, it was evidenced that G $\beta 2$ failed to localize at the mitochondria in MEF Mfn1 ${ }^{-1-}$ cells (Supplementary Fig. S3d) unless Mfn1 was reintroduced into these cells (J.Z., unpublished observation). A fractionation assay further showed that G $\beta 2$ levels were significantly reduced in $\mathrm{Mfn}^{-1-}$ cells (Supplementary Fig. S3e). These observations suggest that the interaction between G $\beta 2$ and Mfn1 may help to regulate their submitochondrial distribution and the mobility of Mfn1, which may be important for determining the fusion or branching at particular submitochondrial regions.

Depletion of G $\beta 2$ decreases mitochondrial fusion. To determine whether endogenous $G \beta 2$ has a regulatory function in mitochondrial fusion, we used G $\beta 2$ shRNA to deplete endogenous G $\beta 2$ (Supplementary Fig. S4a). Interestingly, loss of G $\beta 2$ resulted in a notable change in mitochondrial networking as indicated by mito-green fluorescent protein (GFP) labelling. The mitochondria became small tubules and spheres around the nuclear region, most likely due to impaired mitochondrial fusion (Fig. 5a). The effect of G $\beta 2$ on mitochondria was specific, as reintroduction of G $\beta 2$ into these cells significantly rescued the mitochondrial phenotypes (Fig. 5b,c). Mitochondrial dynamics was analysed by using an assay of visualization and quantification of mitochondrial fusion, based on confocal imaging of cells expressing a mitochondrial matrix-targeted photoactivable GFP (PAGFP) dilution rate ${ }^{29}$. Compared with the fast decrease of mito-PAGFP fluorescence intensity of activated mitochondria in normal HeLa cells observed 30 min after photoactivation, little change or no decrease in mito-PAGFP fluorescence within photoactivated mitochondria in HeLa cells with G 32 shRNA was seen (Fig. $5 \mathrm{~d}-\mathrm{f}$ ), reflecting slower mitochondrial fusion rate. The result thus indicates that mitochondrial fusion was blocked by G 32 shRNA. In addition, overexpression of Flag-G $\beta 2$ significantly caused aggregation of mitochondria around the nucleus in HeLa (Supplementary Fig. S4b, upper panel) and SH-SY5Y cells (not shown), as revealed by confocal microscopy. To further determine that $G \beta 2$ in mitochondria regulates mitochondrial fusion, we constructed mitochondrial targeted G $\beta 2$ using a specific mitochondrial outer membrane targeting signal peptide from $\mathrm{Bcl}-\mathrm{xL}^{30}$. Overexpression of mitochondrial targeted G $\beta 2$ induced profound mitochondrial aggregation at the perinuclear region in HeLa cells. In contrast, mitochondrial targeted G $\gamma 2$ or GFP protein using the same mitochondrial targeting signal had no effect on mitochondrial morphology (Supplementary Fig. S4b, middle and lower panel). Electron microscopy analysis further confirmed that the mitochondria clustered and aggregated around the perinuclear region when Gß2 was ectopically expressed (Supplementary Fig. S4c).

The effects of G及2 on mitochondria are specific. We next determined if the effects of G $\beta 2$ on mitochondria are specific. Overexpression of G $\beta 2$ had little impact on the morphology and distribution of other cellular organelles such as Golgi, lysosomes or endoplasmic reticulum in HeLa cells (Fig. 6a). To determine the effect of G $\beta 2$ on mitochondrial physiology and function, we measured mitochondrial membrane potential in the HeLa cells overexpressing G $\beta 2$ by using TMRM (tetramethylrhodamine, methyl ester) staining and found that there was no significant difference from the parental cells (see Supplementary Fig. S4d). Furthermore, G $\beta 2$ did not seem to have any effect on the cell cycle (see Supplementary Fig. S4e,f).

The surprising finding that $G \beta 2$ has such a regulatory role in controlling mitochondrial fusion prompted us to test if other G $\beta$ subunits and $\mathrm{G} \alpha$ or $\mathrm{G} \gamma$ also have a role in mitochondria. We found 
a

$\begin{array}{llllllll}176 & 240 & 387 & 414 & 592 & 628 & 705 & 735\end{array}$

\begin{tabular}{|c|c|c|c|}
\hline GTPase & cc & TM & $\mathrm{cc}] 1-740(\mathrm{WT})$ \\
\hline GTPase & $c c$ & TM & $\mathrm{cc}] \triangle 241-350$ \\
\hline & $\mathrm{cc}$ & TM & $\mathrm{kc} \mid \triangle 1-387$ \\
\hline
\end{tabular}

b

\begin{tabular}{|c|c|c|c|c|c|c|}
\hline \multirow[b]{2}{*}{ Flag-Gß2 } & \multicolumn{3}{|c|}{ Flow thr. } & \multicolumn{3}{|c|}{ IP } \\
\hline & $+\quad+$ & + & + & + & + & \\
\hline GFP & $+\quad-$ & - & - & + & - & \\
\hline$-(\triangle 1-387)$ & $-\quad+$ & - & - & - & + & \\
\hline $241-350)$ & $-\quad-$ & + & - & - & - & \\
\hline GFP-WT & $-\quad-$ & - & + & & - & \\
\hline Anti-Flag & -- & - & - & & 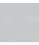 & \\
\hline \multirow{3}{*}{ Anti-GFP } & & & & & & \\
\hline & - & & & & - & \\
\hline & - & & & - & & \\
\hline $\mathrm{HC}$ & & & & & & \\
\hline
\end{tabular}

C

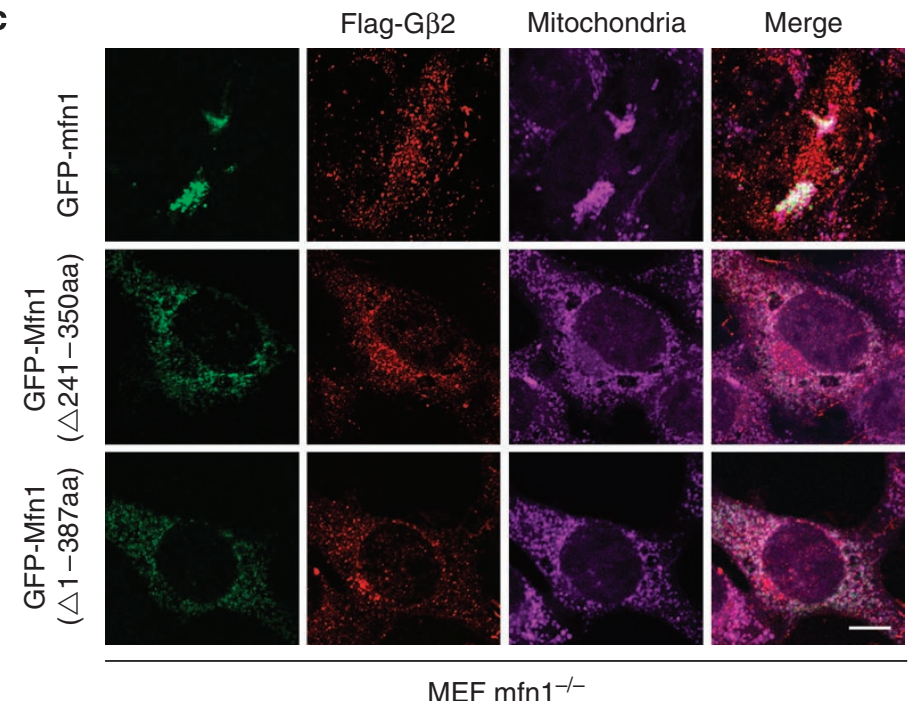

d

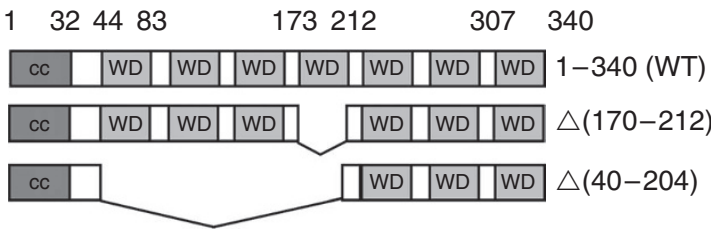

e

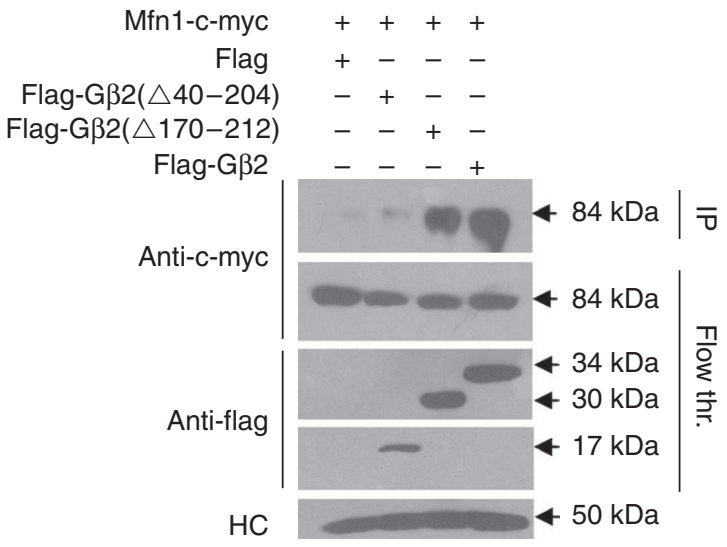

f

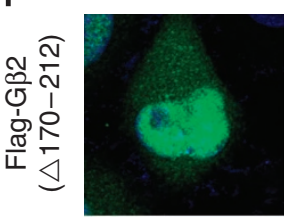

MitoTracker
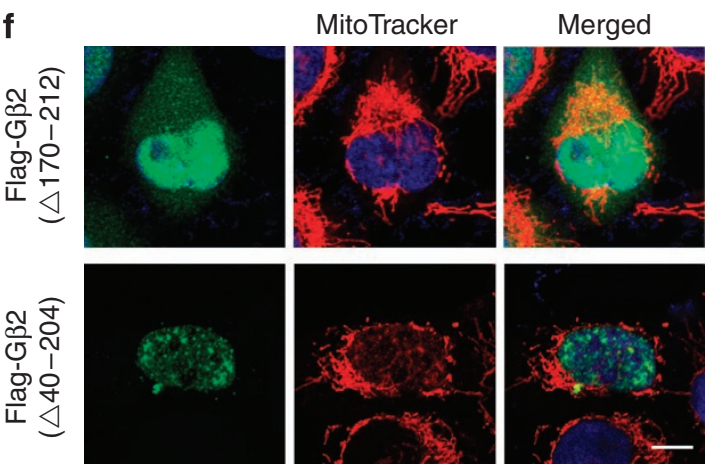

Figure 3 | Identification of the interacting domain between $\mathbf{M f n} \mathbf{1}$ and Gß2. (a) GTPase domain (aa 76-240), two coiled-coil domains (aa 387-414 and 705-735), and transmembrane domain (aa592-628) are indicated as filled grey boxes. A mutant lacking aa 241-350 was named as $\Delta 241-350 ;$ the $\Delta 1-387$ construct contains only C-terminal amino acids from 387 to 740. (b) Cell lysates from the HeLa cells expressing Flag-G $\beta 2$ with either GFP-Mfn1 or GFP-Mfn1 ( $\Delta 241-350)$ or GFP-Mfn1 ( $\Delta 1-387)$ or GFP as indicated were subjected to immunoprecipitation with an antibody against GFP, followed by western blot with a Flag antibody. The heavy chain of immunoglobulins was used as loading control. (c) The confocal images of Mfn1 $1^{-/-}$MEFs expressing the Flag-Gß2 (Red) with either GFP- Mfn1 or GFP-Mfn1 ( $\Delta 241-350)$ or GFP-Mfn1 ( $\Delta 1-387$ ), and mitchondria are visualized with Mitotracker deep red. Scale bar, $10 \mu \mathrm{m}$. (d) Coiled-coil domain (aa1-32) and seven WD40 domains (aa 44-83, 86-125, 132-170, 173-212, 215-254, 257-298 and 307-340) are indicated as filled grey boxes. The mutants lacking the fourth or the first four WD40 domains were named as $\Delta 171-212$ and $\Delta 40-204$, respectively. (e) Lysates either from HeLa cells co-transfected mfn1-c-myc with Flag-G $\beta 2$, Flag-G $\beta 2$ ( $\Delta 171-212$ ), Flag-G $\beta 2$ ( $\Delta 40-204)$ or Flag as indicated were subjected to IP with an antibody against Flag, followed by western blots with a c-myc antibody. (f) The representative images of the HeLa cells expressing the Flag-G $\beta 2(\Delta 171-212)$ or Flag- G $\beta 2(\Delta 40-204)$ (green) and mitochondria were visualized with $20 \mathrm{nM}$ MitoTracker Red.

that G $\beta 1$ induced a small fraction (10\%) of cells with mitochondrial aggregation, whereas other G $\beta$ proteins failed to alter mitochondrial morphology (Fig. 6b). RACK1, a protein also named G $\beta 2$ like protein 1 with WD40 repeat domains, could not induce mitochondrial aggregation in the cells (Fig. 6b). G $\gamma 2$ or its mutant form (C68S), which fails to localize at the membrane, did neither affect mitochondrial morphology nor the distribution of G $\beta 2$ (Fig. $6 c, d$ ).

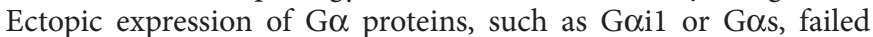
to induce the alteration of mitochondrial morphology or restore dysfunctioned network of mitochondria induced by G 32 knockdown (Fig. 7a). Mitochondrial aggregation was evident when cells

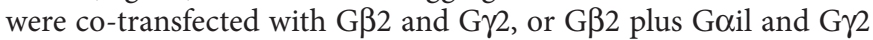
(Fig. 7a,b), although the overall percentage of transfected cells with aggregated mitochondria was slightly reduced compared with

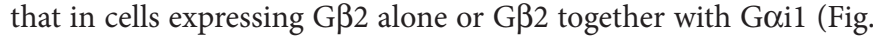
$7 \mathrm{c})$. G $\beta 2$ lacking the $\mathrm{G} \gamma$ interacting domain is still able to induce the mitochondrial aggregation; even when it is coexpressed with $\mathrm{G} \gamma 2$. Moreover, ectopic expression of $\mathrm{G} \gamma 2$ failed to restore fragmented mitochondria induced by G $\beta 2$ knockdown (Fig. 7d-f). Taken together, we demonstrate that the effect of G $\beta 2$ on mitochondria is unique and G $\gamma 2$ has limited effect on the regulatory role of G $\beta 2$ on mitochondrial fusion.

\section{Discussion}

Our results have demonstrated a novel role of G 32 in Mfn1dependent mitochondrial fusion. We found that mitochondrial Gß2 
a
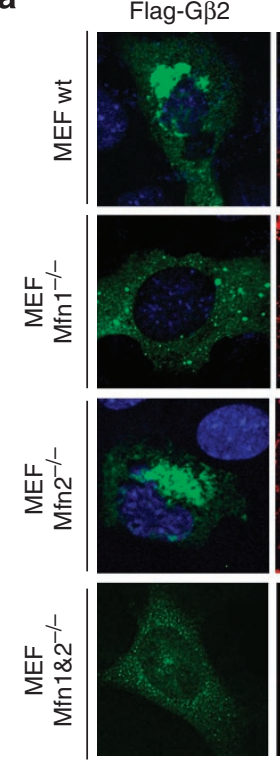

d

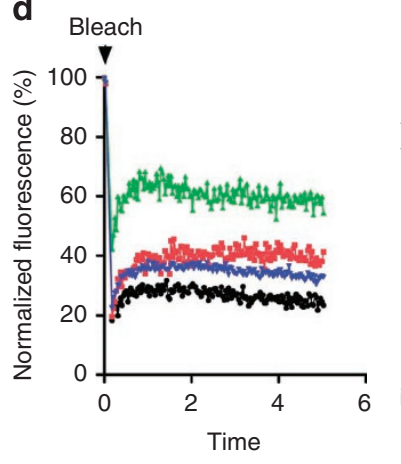

MitoTracker
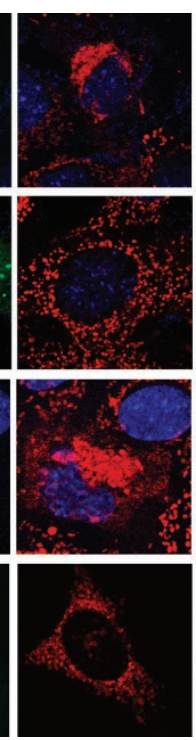

e

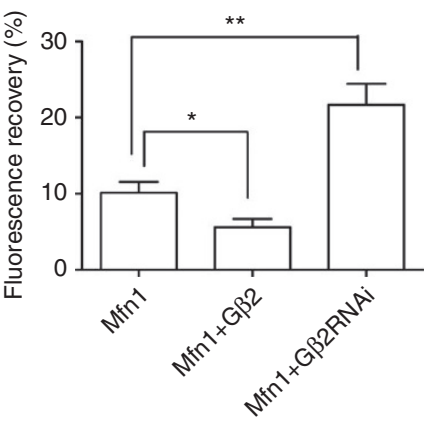

b
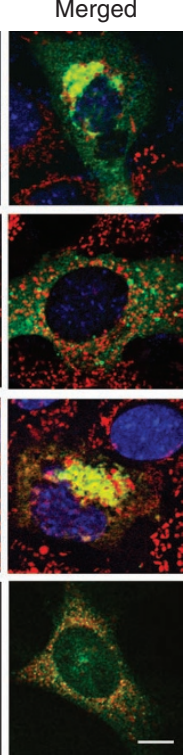

c
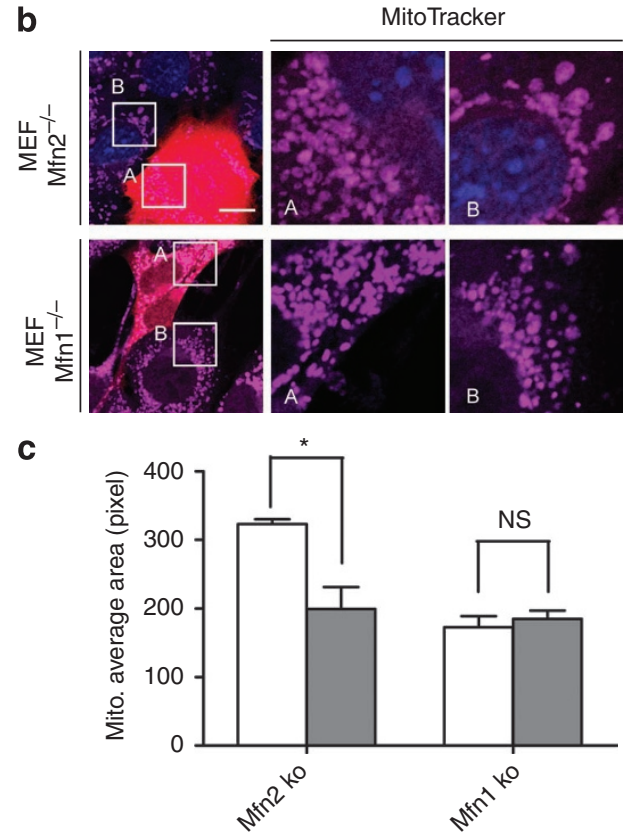

f

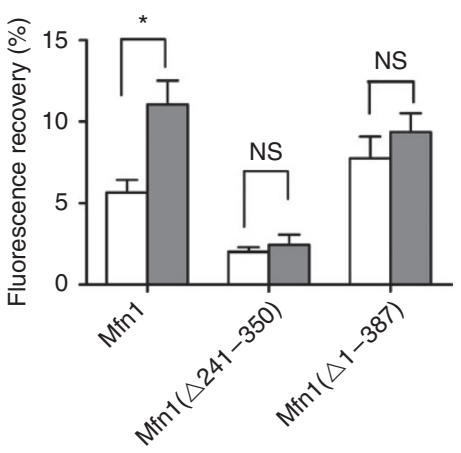

Figure 4 | Gß2 requires Mfn1 for its effect on mitochondrial dynamics. (a) Representative images of wild type or Mfn1 ${ }^{-/-}$and/or Mfn2 ${ }^{-/-}$MEF cells transfected with Flag-tagged G 32 . Mitochondria in cells were stained with MitoTracker Red and Flag- G $\beta 2$ were immunostained with a Flag-specific antibody. Scale bar, $10 \mu \mathrm{m}$. (b) Mitochondria in $\mathrm{Mfn}^{2^{-/}}$cells, and $\mathrm{Mfn}^{-/-}$cells are shown in the representative images of $\mathrm{Mfn}^{-/-}$or Mfn2 ${ }^{-/-} \mathrm{MEFs}$ transfected with G $\beta 2$ shRNA (red). The box area A (tranfected cell) and B (untransfectd cell) in the left panel show the different mitochondrial size at higher magnifications. Scale bar, $10 \mu \mathrm{m}$. (c) The quantification of mitochondrial size scored by Image J (Wayne Rasband, NIH). Filled box represents mitochondrial size in Mfn2 ${ }^{-/-}$MEFs and open box represents in Mfn2 ${ }^{-/-}$MEFs transfected with G $\beta 2$ shRNA. The data represent the mean s.d., $n=10-15$. Differences in the mean mitochondrial average area between $\mathrm{mfn} 2^{-/-} \mathrm{MEFs}$ and $\mathrm{mfn} 2^{-/-}$MEFs with G $\beta 2$ shRNA were statistically significant at ${ }^{\star} P<0.05$.

(d) Mfn1-YFP was co-transfected into HeLa cells with either Flag- G $\beta 2(\bullet$, black) or G $\beta 2$ shRNA ( $\boldsymbol{\square}$, red) and the mitochondrial Mfn1 mobility was measured by FRAP. Fis1 ( $\boldsymbol{\Delta}$, green) was used as a control of fast membrane mobility as previously reported ${ }^{28}$ and Mfn1 alone ( $\boldsymbol{\nabla}$, blue) was used as baseline control. Each line represents the mean of $\geq 20$ measurements. (e) The fluorescence recovery of Mfn1-YFP in HeLa cells and the cells co-transfected with either Flag-G $\beta 2$ or G 32 shRNA was measured by FRAP. Differences in the mean fluorescence recovery between Mfn1-YFP in HeLa cells and Mfn1-YFP in HeLa cells co-transfected with Flag-G $\beta 2$, or with G 32 shRNA were statistically significant ${ }^{\star} P<0.05$ and ${ }^{\star \star} P<0.005$; Error bars represent the s.e.m., $n=21$. (f) Mobility of Mfn1-GFP, or Mfn1-GFP ( $\triangle 241-350)$, Mfn1-GFP ( $\triangle 1-387)$ in HeLa cells and the cells co-transfected with DsRed-G $\beta 2$ shRNA was measured by FRAP. The fluorescence recovery of Mfn1-GFP, Mfn1-GFP ( $\Delta 241-350)$ and Mfn1-GFP ( $\Delta 1-387)$ in HeLa cells and the cells co-transfected with G $\beta 2$ shRNA was measured by FRAP. ${ }^{\star} P<0.05$ and NS means nonsignificant. Data represent the mean s.e.m., $n=20$.

interacts with Mfn1 and regulates the mobility of Mfn1. It is possible that $\mathrm{G} \beta 2$, as a scaffold protein $^{31}$, may function to mediate the clustering of Mfn 1 molecules at the microregion of the mitochondrial outer membrane. As a result, G $\beta 2$ may modulate the distribution and activity of Mfn1, which could be important in mitochondrial fusion or branching at specific submitochondrial sites. Intriguingly, several WD40 proteins were found to interact with Dnm1 (Drp1 homologue in yeast), a key protein in mediating fission, for mitochondrial fis$\operatorname{sion}^{13,32}$ and Caf4 regulates the polarized distribution of Dnm $1^{12}$. Thus, it is of great interest to note that distinct proteins with WD40 repeat domains are involved in both mitochondrial fusion and fission by regulating the submitochondrial distribution of proteins for these processes.

On the plasma membrane, G $\beta \gamma$ interacts with $\mathrm{G} \alpha$ and subsequently transduces signals to the downstream effectors. We found that G-protein-coupled receptor activators (ET1, PGE2) or antagonists (PTX), which are known to affect the dissociation/association of $\mathrm{G} \alpha$ and $\mathrm{G} \beta \gamma$, did not have any effect on mitochondrial networking or G $\beta 2$-induced changes in mitochondrial networking (J.Z., unpublished observations). These data do not support that 
a
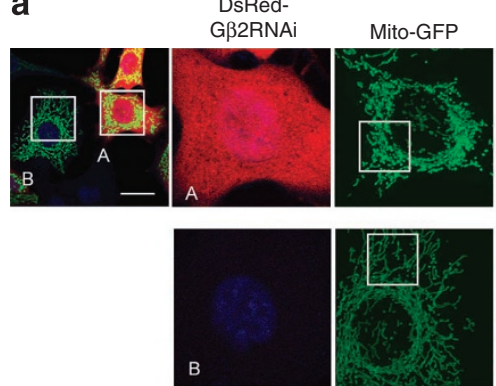
Zoom
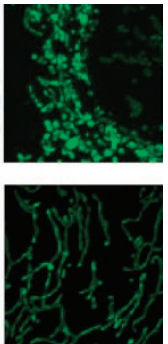

b

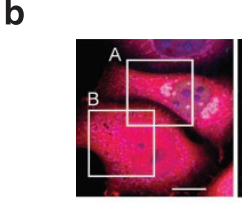

Flag-Gß2*

MitoTracker

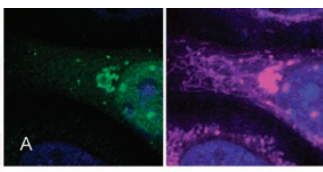

d

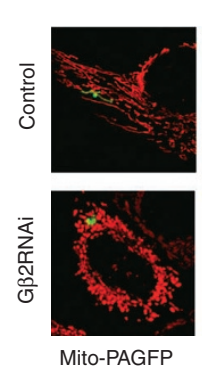

$0 \mathrm{~min}$

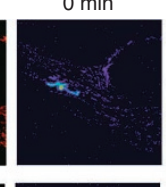

$10 \mathrm{~min}$

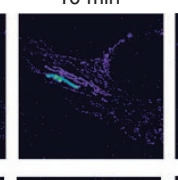

Pseudocoloured
C
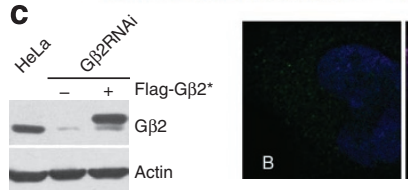

$20 \min$

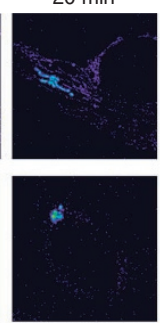

$30 \mathrm{~min}$
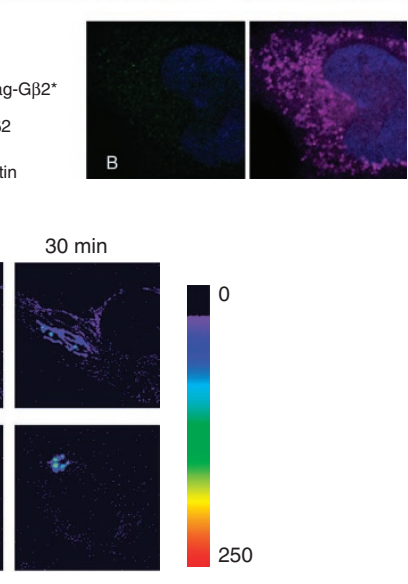

50

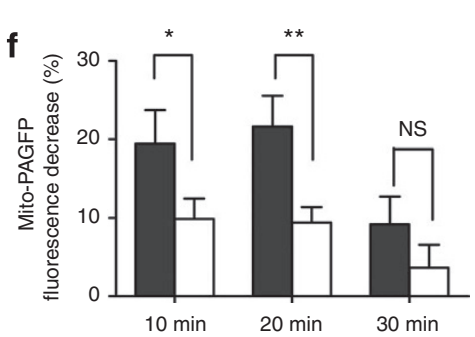

Figure $\mathbf{5}$ | G $\beta 2$ has a regulatory role in mitochondrial morphology. (a) HeLa cells were co-transfected with DsRed-G $\beta 2$ shRNA (red) and mitochondrial matrix localized GFP (mito-GFP), and the expression of G 32 shRNA (box A) and mitochondrial morphology was visualized by confocal microscopy (left panel). Scale bar, $10 \mu \mathrm{m}$. (b) GB2 shRNA HeLa cells (red) were transfected with a mutant of Flag-G $\beta 2$ (green) as described in Figure 2. The cells were stained with $10 \mathrm{nM}$ Mitotracker Deep Red for visualizing mitochondria. Scale bar, $10 \mu \mathrm{m}$. (c) The G $\beta 2$ protein levels in the cells were determined by western blot: HeLa cells (left lane), HeLa cells with either G 32 shRNA (middle lane) or G $\beta 2$ shRNA with reintroduced mutant of Flag-G $\beta 2$ (right lane). (d) G $\beta 2$ RNAi block mitochondrial fusion. HeLa cells and G $\beta 2$ shRNA stable cells were transfected with mito-PAGFP and followed by photoactivation. Images were collected every $10 \mathrm{~min}$ over $30 \mathrm{~min}$. Representative images shown are pseudo-coloured projections of z-series to represent changes in the fluorescence intensities. The fluorescence intensity of mito-PAGFP is highlighted in the pseudocoloured images where fusion of photoactivated 'orange' mitochondria with nonactivated 'purple' organelles is followed by the formation of the 'yellow/blue' intermediates. (e) Normalized fluorescence intensity of mito-PAGFP in HeLa cells (black) and G $\beta 2$ shRNA stable cells (red) at each time point was measured and plotted against time. Each experimental group contains at least 20 cells. (f) Values of percent mito-PAGFP fluorescence in HeLa cells and G 32 shRNA stable cells decrease after 10, 20, and 30 min in each experimental group, and were plotted against time. Filled box represents G $\beta 2$ shRNA cells and open box represents normal HeLa cells. The error bars represent s.e.m., $n=26$ cell time-lapse measurements per group. Differences in the mean fluorescence decrease percentage between HeLa cells and HeLa cells with G $\beta 2$ shRNA in 10 min and in 20 min were statistically significant ${ }^{\star} P<0.05$ and ${ }^{\star \star} P<0.005$, two-tailed $t$-test.

the activation of G-protein-coupled receptor signalling pathway or the translocation of $\mathrm{G} \beta \gamma$ from the plasma membrane onto mitochondria are important for the altered mitochondrial networking

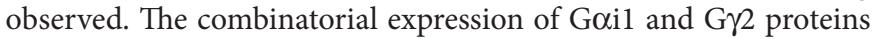
has limited effects on G $\beta 2$-induced mitochondrial aggregation (see Fig. 7). Mutant G 2 -C68S, which fails to be modified by lipid and fails to localize to mitochondria, also did not affect mitochondrial morphology induced by enforced expression of G $\beta 2$. Recently, it

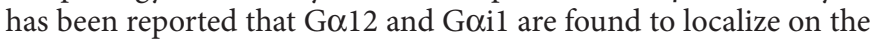
surface of mitochondria ${ }^{23,24}$. However, their role in mitochondria is largely unknown. We detected a weak interaction between G $\beta 2$ with

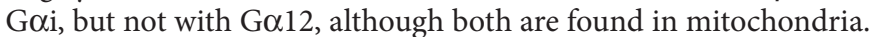
More studies are needed to further dissect the significant role of $G$ proteins in regulating mitochondrial phenotypes and the interplay between mitochondria and other cellular events.

Previous studies have also shown that G $\beta \gamma$ interacts with dynein and tubulin ${ }^{33}$ to regulate microtubule reorganization and cell polarity ${ }^{18,19}$. Indeed, we have found that $\mathrm{G} \beta 2$ was able to interact with tubulin in co-immunoprecipitation analysis (data not shown). A G $\beta$ protein was also found to interact with dynein intermediate chain, a member in the dynein family ${ }^{31}$. The interaction of G $\beta 2$ with microtubules or motor proteins may provide an additional force for bringing mitochondria in proximity for mitochondrial tethering and docking, leading to an initiation of fusion.

\section{Methods}

Plasmid construction and mutagenesis. The human Mfn1 cDNA obtained from Dr Margaret Fuller (Stanford University, USA) was subcloned into a plasmid vector pECFP-N1 (Clontech) using EcoRI and BamHI. The Gß2 gene was cloned by PCR from a human fetal liver CDNA library, and the G 32 CDNA was inserted into plasmid vectors pFLAG-CMV-4 (Sigma) and pEYFP-C1 (Clontech) to generate pFLAG-Gß2 and pEYFP-GB2 using HindIII and EcoRI, respectively. The cDNA fragment encoding the Bcl-xL mitochondrial target peptide was amplified by PCR and fused to the $C$ terminal of $G \beta 2$ in pFLAG-G $\beta 2$, named as Flag-G $\beta 2-M$. The $\mathrm{GFP}_{10}$-GNG2 and pEGFP-GNB1 plasmids were from Dr Terence E. Hébert (McGill 
a

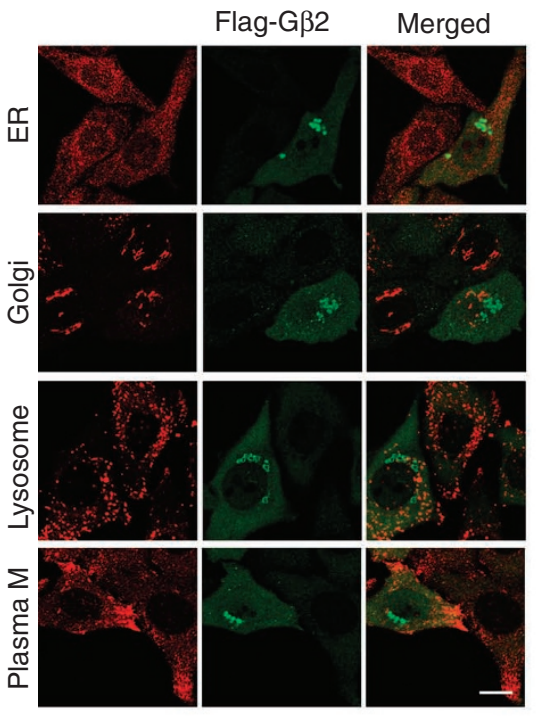

b

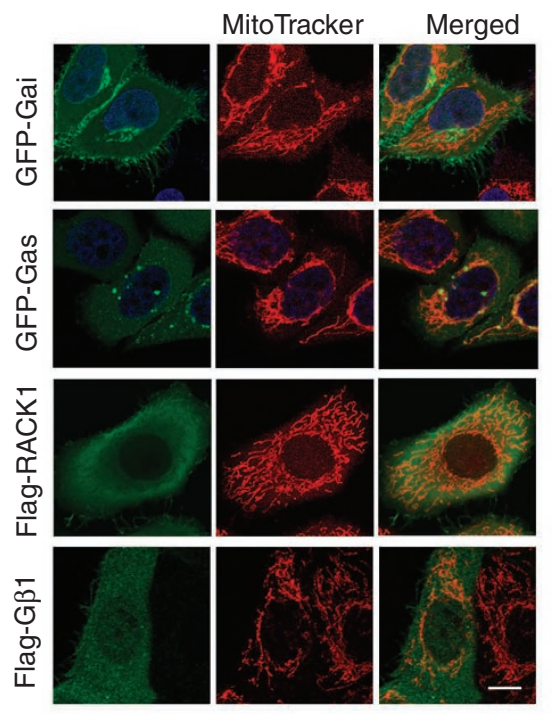

C

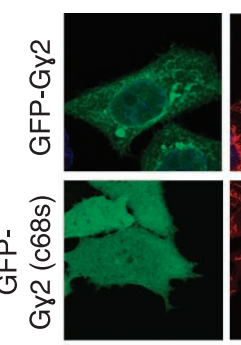

MitoTracker

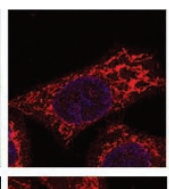

Merged

d

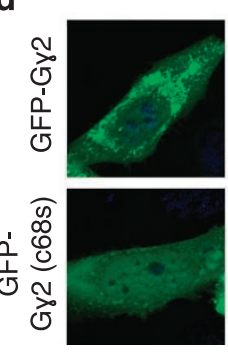

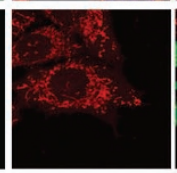

Flag-G $\beta 2$

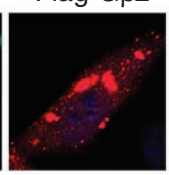

Merged

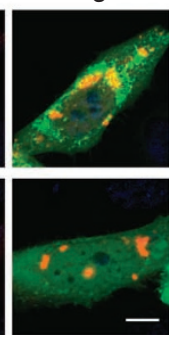

Figure 6 | The effects of $\mathbf{G} \boldsymbol{\beta 2}$ on mitochondria are specific. (a) Representative images of morphology of endoplasmic reticulum, lysosomes, Golgi, plasma membrane in HeLa cells with enforced expression of Flag-G 32 . HeLa cells were transfected with Flag-G 32 . After incubation for $24 \mathrm{~h}$, the cells were fixed in 4\% paraformadehyde and double immunostained Flag (green) with either of the antibodies: Calnexin, GM130, Lamp3 or Transferrin receptor

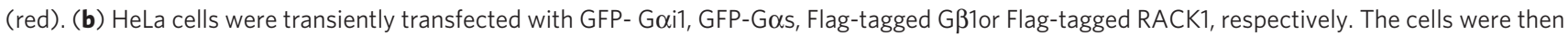
loaded with $20 \mathrm{nM}$ MitoTracker Red for visualizing mitochondria, and immunostained Flag (green) for labelling Flag-tagged G $\beta 1$ lor Flag-tagged RACK1. (c) Confocal images of HeLa cells transfected with GFP-G 22 or G 2 (C68S). After incubation for 24h, the cells were stained with Mitotracker (red). (d) HeLa cells co-transfected Flag-G $\beta 2$ with GFP-G $\gamma 2$ or G $\gamma 2$ (C68S), and the cell were fixed and immunostained with Flag antibody (red). Sale bar, $10 \mu \mathrm{m}$.

University, Canada). The G 72 cDNA was subcloned to pFLAG-CMV-4 and the mutant (C68S) of $\mathrm{GFP}_{10}-\mathrm{G} \gamma 2$ was generated by site-directed mutagenesis. Flag-G $\gamma 2$ $\mathrm{M}$ was generated as described above. The GNB1 cDNA was subcloned to pEYFP-C1 and pFLAG-CMV-4 using HindIII and KpnI, respectively. The cDNA fragment encoding the mitochondrial matrix-localized signal peptide of TRX2 was subcloned to pEYFP-C1 and pEGFP-C1 using EcoRI and BamHI. The QuickChange sitedirected mutagenesis kit was purchased from Stratagene. The GNB2 RNAi target sequences: CCTGGATGACAACCAAATC were designed by company (RuiBo) and subcloned to the RNAi-Ready pSIREN-RetroQ-DsRed-Express Vector (Clontech).

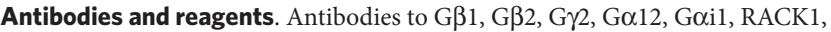
c-Myc, VDAC1 and LAMP3 were purchased from Santa Cruz, and the concentrations of them are $200 \mu \mathrm{g} \mathrm{ml}^{-1}$. Antibodies to Flag and $\alpha$-tubulin were from Sigma, and the concentrations of both are $1 \mathrm{mg} \mathrm{ml}^{-1}$. Antibodies to Tom20, Tim23, GM130, Transferrin receptor and Calnexin were from Becton Dickinson, and the concentrations of them are $1 \mathrm{mg} \mathrm{ml}^{-1}$. Antibodies to Mfn1 and Mfn2 monoclonal were from Novus, the concentrations of both are $500 \mu \mathrm{g} \mathrm{ml}^{-1}$. The antibodies were diluted 1:100 of the original concentration for immunostaining and diluted 1:1,000-1:5,000 for western blotting. Mitotracker Red and Mitotracker Deep Red were purchased from Invitrogen.

Cell culture and transfections. Mfn1 and Mfn2 knockout, and Mfn1 and Mfn2 double knockout MEF cells were kind gifts from Dr David Chan (California Institute of Technology). HeLa and SH-SY5Y cells (American Type Culture Collection) were maintained in Dulbecco's modified Eagle's medium with $10 \%$ fetal bovine serum, $100 \mathrm{U} \mathrm{ml}^{-1}$ penicillin and $100 \mathrm{U} \mathrm{ml}^{-1}$ streptomycin at $37^{\circ} \mathrm{C}$ in a $5 \%$ $\mathrm{CO}_{2}$ incubator. Cells were transfected with plasmid DNAs by using a phosphate calcium method.

Confocal microscopy. HeLa and SH-SY5Y cells cultured on gelatin-coated coverslips were fixed with $3.7 \%$ paraformaldehyde, followed by permeabilization in $0.5 \%$ Triton X-100. Cells were incubated with primary antibodies in phosphatebuffered saline (PBS) supplemented with $5 \%$ bovine serum albumin, followed by incubation with appropriate secondary antibodies. To visualize mitochondria in living cells, cells were incubated with either MitoTracker Deep Red 633 or Red CMXRos (Invitrogen) at $37^{\circ} \mathrm{C}$ for $30 \mathrm{~min}$ and washed twice with PBS. Images were visualized under an LSM 510 confocal microscope (Carl Zeiss), equipped with $63 \times / 1.45$ oil objective and processed using LSM Browser (Carl Zeiss) and Photoshop (Adobe).

Mitochondria fractionation. The mitochondrial membrane was purified on discontinuous sucrose gradients as previously described ${ }^{34}$, with some modifications.
Briefly, cells were washed twice in PBS and resuspended in $1.5 \mathrm{ml}$ buffer A $(20 \mathrm{mM}$ Tricine, $250 \mathrm{mM}$ sucrose) containing protease inhibitors for $20 \mathrm{~min}$ on ice and then subjected to homogenization. After 20 strokes, the cell homogenate was collected and $100 \mu \mathrm{l}$ of the samples were taken out as whole cell lysate (W). The remaining cell homogenate was centrifuged at $1,000 \times g$ for $8 \mathrm{~min}$ at $4{ }^{\circ} \mathrm{C}$, and the supernatant was as the postnuclear solution (PNS). PNS (100 $\mu \mathrm{l})$ was centrifuged at $10,000 \times g$, and the supernant was collected as C2. PNS ( $2 \mathrm{ml})$ was layered on a Percoll gradient $(30 \%)$ and centrifuged at $30,000 \times g$ for $30 \mathrm{~min}$ at $4{ }^{\circ} \mathrm{C}$, a white band $(1-2 \mathrm{ml})$ at $2 \mathrm{~cm}$ in Percoll gradient just below the PNS was collected as the plasma membrane fraction (P). P was continuingly centrifuged at $100,000 \times g$ for $1 \mathrm{~h}$ at $4^{\circ} \mathrm{C}$ to remove the percoll. Another PNS $(2 \mathrm{ml})$ was centrifuged at $10,000 \times g$, and the pellet was washed twice with Buffer B ( $0.225 \mathrm{M}$ mannitol, $1 \mathrm{mM}$ EGTA, $25 \mathrm{mM}$ HEPES, pH 7.4) and resuspended in $0.5 \mathrm{ml}$ Buffer B. The suspension was layered to a Percoll density gradient $(2 \mathrm{ml} 80 \%, 4.5 \mathrm{ml} \mathrm{52 \%}$ and $4.25 \mathrm{ml} \mathrm{26 \%})$ and centrifuged at $100,000 \times g$ for $1 \mathrm{~h}$ at $4^{\circ} \mathrm{C}$. The band between the Percoll $26 \%$ and $52 \%$ gradient was collected as the pure mitochondrial fractionation (M). C1 was the supernatant obtained after the cell homogenate was centrifuged at $250,000 \times g$ for $1 \mathrm{~h}$ at $4^{\circ} \mathrm{C}$.

Mitochondria membrane motility assay. The mitochondria membrane motility was measured as previous described ${ }^{28}$. Briefly, circular region of interests (ROIs), $2.5 \mathrm{~mm}$ in diameter, were imaged (a-Plan-FLUAR $100 \times / 1.45$ oil objective; Carl Zeiss) before and after photobleaching (20 iterations of 514-nm laser (488-nm laser for GFP) set to $100 \%$ ) of 0.5 -mm circular ROIs located in the centre of imaging area. Totally, 150 images ( 1 scan every $33 \mathrm{~ms}$ ) were collected and the fluorescence intensity was digitalized in imaged ROIs with an LSM 510 software (Zeiss MicroImaging).

FRET assay. All FRET observations were performed under a Leica DM IRE2 confocal laser scanning microscope, after cells were transfected for $24 \mathrm{~h}$. The donor (CFP) was excited at $458 \mathrm{~nm}$, and its fluorescence was detected in a wavelength of $478-498 \mathrm{~nm}$ (CFP channel), whereas the excitation at $514 \mathrm{~nm}$ and the emission at $545 \pm 15 \mathrm{~nm}$ were used for detecting the acceptor (YFP) (YFP channel). FRET was detected at the excitation of $458 \mathrm{~nm}$ and the emission of $545 \pm 15 \mathrm{~nm}$ (FRET channel). Fluorescence images of the transfected cells were taken at the CFP-, YFP- and FRET channels sequentially. Dequenching of the donor fluorescence by photobleaching of the acceptor YFP was performed by illuminating the transfected cells at the YFP excitation wavelength $(514 \mathrm{~nm})$ for 250 iterations, and then the CFP-Mfn 1 images were taken at the same focal plane. The FRET efficiency was calculated using the equation: $E=1-\left(F_{\mathrm{DA}} / F_{\mathrm{D}}\right)$, where $F_{\mathrm{DA}}$ and $F_{\mathrm{D}}$ are the fluorescence intensity of CFP in the cells expressing both donor and acceptor, and donor alone (acceptor was quenched), respectively ${ }^{26}$. 
a
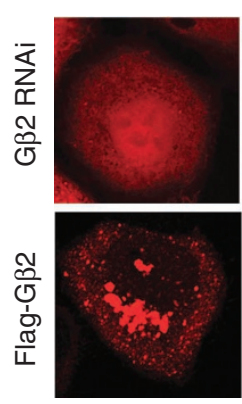

b

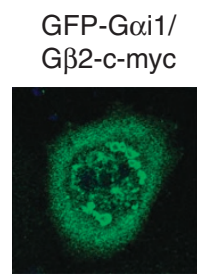

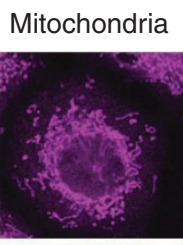
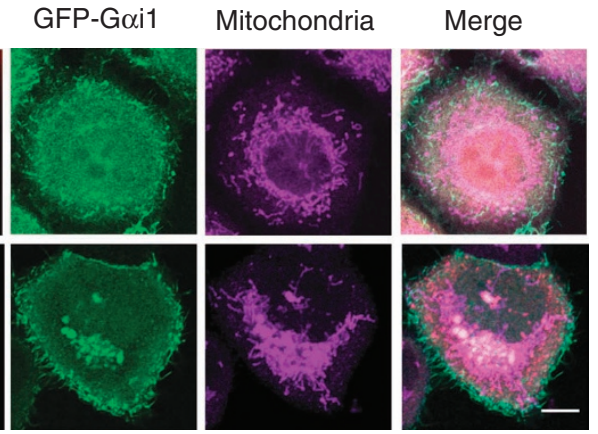

d

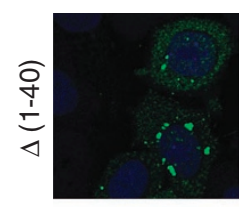

Gy2
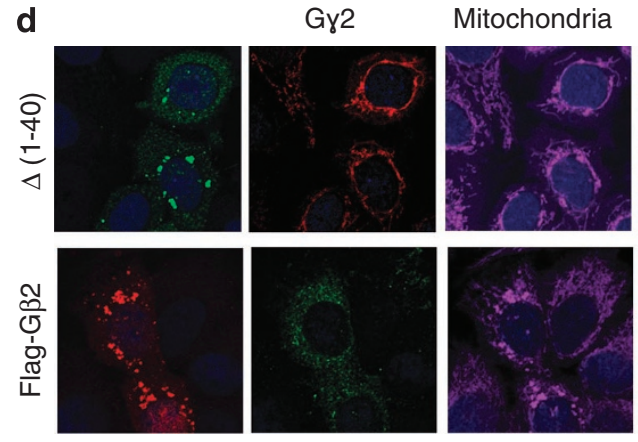

e
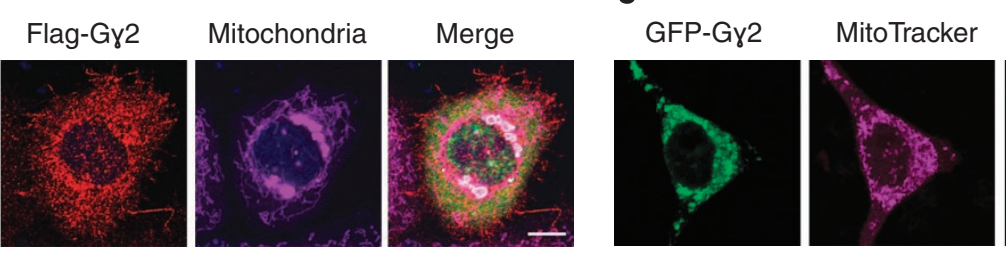

DsRed-
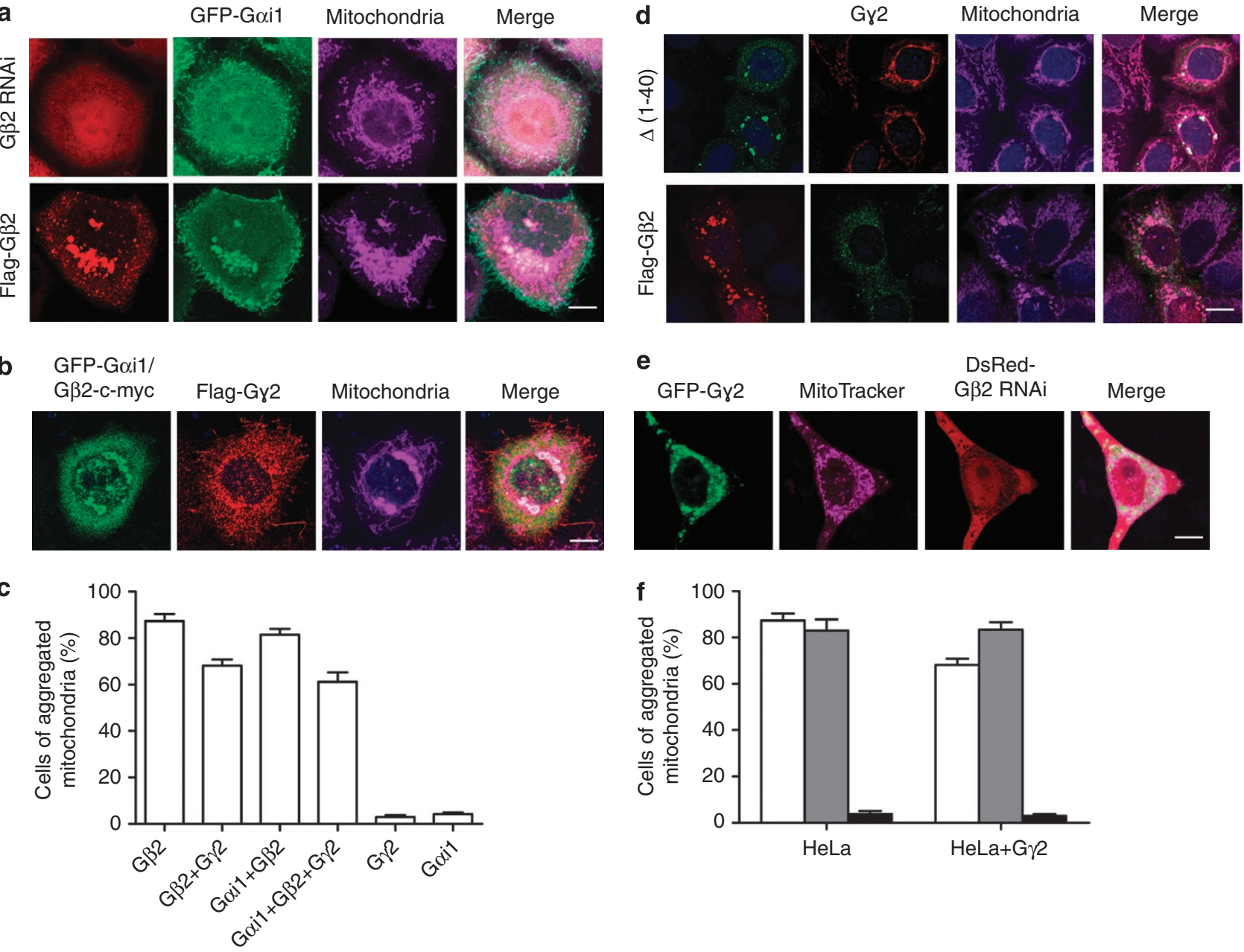

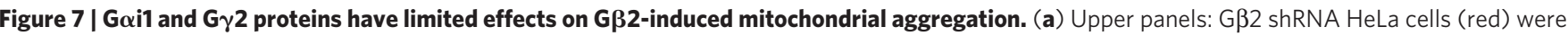
transfected with GFP-Goil (green) and loaded with 20 nM MitoTracker Deep Red; bottom panels: HeLa cells were co-transfected with GFP-Goil and Flag-G 32 (red) and loaded with 20 nM MitoTracker Deep Red. (b) HeLa cells were transfected with Flag-G $\gamma 2$, G $\beta 2-c-m y c$ and YFP-G $\alpha$ i1. After incubation for $24 \mathrm{~h}$, the cells were incubated with Mitotracker (Deep Red), fixed and immunostained with Flag antibody (red) and c-myc antibody (green). (c) The histogram indicates the mitochondrial aggregation percentage in the cells transfected with either of the coupled pairs: Flag-G $\beta 2$ alone, Flag-G $\beta 2$ and

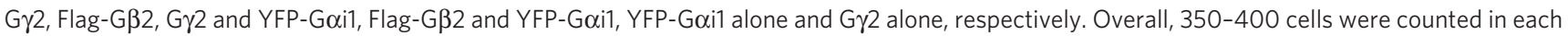
experiment. Error bars represent the s.e.m. (d) HeLa cells were transfected with G $\gamma 2$ and Flag-G $\beta 2$ or Flag-G $\beta 2$ ( $\Delta 1-40)$. After incubation for 24 h, the cells were incubated with Mitotracker (Deep Red), fixed and immunostained with Flag antibody and G $\gamma 2$ antibody. (e) G $\beta 2$ shRNA HeLa cells (red) were transfected with Flag-G $\gamma 2$ (green) and loaded with $20 \mathrm{nM}$ MitoTracker Deep Red. The scale bar in all images represents $10 \mu \mathrm{m}$. (f) The histogram indicates the mitochondrial aggregation percentage in the cells transfected with either of the coupled pairs: Flag-G $\beta 2$ alone (open box), Flag-G $\beta 2$ ( $\Delta 1-40$ ) (grey box), Flag-G $\beta 2$ and $G \gamma 2$ (open box), Flag-G $\beta 2(\Delta 1-40$ ) and $G \gamma 2$ (grey box), G $\gamma 2$ alone and null vector (filled box), respectively. Error bars represent the s.e.m. Quantification was carried out as described in c.

Immunoprecipitation. Cells were collected and washed twice with PBS, and then resuspended in lysis buffer $(0.75 \mathrm{M}$ aminocaproic acid, $50 \mathrm{mM}$ Bis-Tris, $\mathrm{pH} 7.0$, $1.5 \% n$-dodecyl-b-d-maltopyranoside, $1 \mathrm{mM}$ phenylmethyl sulphonyl fluoride, $1 \mathrm{mg} \mathrm{ml}^{-1}$ leutpeptin and $1 \mathrm{mg} \mathrm{ml}^{-1}$ pepstatin) for $30 \mathrm{~min}$ on ice, and, then subjected to centrifugation at $72,000 \mathrm{~g}$ for $20 \mathrm{~min}$ at $4^{\circ} \mathrm{C}$. The primary monoclonal antibody $(2 \mu \mathrm{g})$ was added into the supernatant containing $500 \mu \mathrm{g}$ proteins, and incubated at $4{ }^{\circ} \mathrm{C}$ overnight. After incubation with Protein G-agarose (Santa Cruz Biotech) for $2 \mathrm{~h}$ at $4^{\circ} \mathrm{C}$, the beads were washed three times with the lysis buffer and boiled with $6 \times$ loading buffer for $5 \mathrm{~min}$. The proteins were then separated by SDS-polyacrylamide gel electrophoresis, and western blotting was performed with proper antibodies as indicated.

Mitochondrial membrane potential assay. The mitochondrial membrane potential was measured. Briefly, HeLa cells were transfected with an empty vector and pFlag-G 32 . After $24 \mathrm{~h}$, the cells were collected and stained with $200 \mathrm{nM}$ TMRM (Invitrogen) for $15 \mathrm{~min}$ at room temperature, and then kept at $4^{\circ} \mathrm{C}$ for measurement after washing once with PBS. For each sample, 50,000 cells were scored after excitation with a $488 \mathrm{~nm}$ argon laser and emission through the phycoerythrin filter $(575 \mathrm{~nm})$ of a flow cytometer (Becton Dickinson). The percentile of cells above and below the threshold TMRM fluorescence signal was determined with Cellquest software.

Cell cycle analysis. Cells were collected and washed and then resuspended in PBS. The cell suspension containing $1 \times 10^{6}$ cells in $500 \mu \mathrm{l}$ was fixed by adding $5 \mathrm{ml}$ cold $\left(-20^{\circ} \mathrm{C}\right) 75 \%$ ethanol at $4^{\circ} \mathrm{C}$ overnight. The cells were centrifuged at $1,000 \times \mathrm{g}$ for $10 \mathrm{~min}$ at $4^{\circ} \mathrm{C}$ and washed twice with $5 \mathrm{ml}$ PBS plus $1 \%$ bovine serum albumin, and resuspended in $400 \mu \mathrm{l}$ PBS supplemented with $1 \%$ bovine serum albumin, $50 \mu \mathrm{l}$ $500 \mu \mathrm{g} \mathrm{ml}^{-1}$ propidium iodide and $50 \mu \mathrm{l}$ boiled RNase A $\left(10 \mathrm{mg} \mathrm{ml}^{-1}\right)$. After incubating at $37^{\circ} \mathrm{C}$ for $30 \mathrm{~min}$, the cells were analysed by FACS using an FL2 histogram at $620 \mathrm{~nm}$ (Becton Dickinson).

Photoactivation assay. Cells were grown in two-well chambers for confocal microscopy. Images were captured with a microscope (model LSM 510; Carl Zeiss MicroImaging) using a $63 \times 1.4$ NA Apochromat objective (Carl Zeiss MicroImaging). Light (405- or 413-nm) was used for photoactivation of PAGFP. ROIs were selected and series of $z$-sections from the top to the cell bottom with intervals between sections set to $0.5-0.75 \mu \mathrm{m}$ were irradiated with 405 - or 413 -nm light. The same intervals between optical sections were used for imaging. 


\section{References}

1. Liu, C. Y., Lee, C. F., Hong, C. H. \& Wei, Y. H. Mitochondrial DNA mutation and depletion increase the susceptibility of human cells to apoptosis. Ann. N Y Acad. Sci. 1011, 133-145 (2004).

2. Youle, R. J. \& Karbowski, M. Mitochondrial fission in apoptosis. Nat. Rev. Mol. Cell Biol. 6, 657-663 (2005).

3. Baloh, R. H., Schmidt, R. E., Pestronk, A. \& Milbrandt, J. Altered axonal mitochondrial transport in the pathogenesis of Charcot-Marie-Tooth disease from mitofusin 2 mutations. J. Neurosci. 27, 422-430 (2007).

4. Chen, H. \& Chan, D. C. Mitochondrial dynamics-fusion, fission, movement, and mitophagy-in neurodegenerative diseases. Hum. Mol. Genet. 18, R169-R176 (2009)

5. Danino, D. \& Hinshaw, J. E. Dynamin family of mechanoenzymes. Curr. Opin. Cell Biol. 13, 454-460 (2001).

6. Chan, D. C. Dissecting mitochondrial fusion. Dev. Cell 11, 592-594 (2006).

7. Bereiter-Hahn, J. \& Voth, M. Dynamics of mitochondria in living cells: shape changes, dislocations, fusion, and fission of mitochondria. Microsc. Res. Tech. 27, 198-219 (1994).

8. Yaffe, M. P. Dynamic mitochondria. Nat. Cell Biol. 1, E149-E150 (1999).

9. Kulski, J. K. et al. Gene expression profiling of Japanese psoriatic skin reveals an increased activity in molecular stress and immune response signals. J. Mol. Med. 83, 964-975 (2005).

10. Hoppins, S., Lackner, L. \& Nunnari, J. The machines that divide and fuse mitochondria. Annu. Rev. Biochem. 76, 751-780 (2007).

11. Nunnari, J. et al. Mitochondrial transmission during mating in Saccharomyces cerevisiae is determined by mitochondrial fusion and fission and the intramitochondrial segregation of mitochondrial DNA. Mol. Biol. Cell $\mathbf{8}$, 1233-1242 (1997)

12. Schauss, A. C., Bewersdorf, J. \& Jakobs, S. Fislp and Caf4p, but not Mdvlp, determine the polar localization of Dnm1p clusters on the mitochondrial surface. J. Cell Sci. 119, 3098-3106 (2006).

13. Griffin, E. E., Graumann, J. \& Chan, D. C. The WD40 protein Caf4p is a component of the mitochondrial fission machinery and recruits Dnm1p to mitochondria. J. Cell Biol. 170, 237-248 (2005)

14. Ishihara, N., Eura, Y. \& Mihara, K. Mitofusin 1 and 2 play distinct roles in mitochondrial fusion reactions via GTPase activity. J. Cell Sci. 117, 6535-6546 (2004).

15. Koshiba, T. et al. Structural basis of mitochondrial tethering by mitofusin complexes. Science 305, 858-862 (2004).

16. Sondek, J., Bohm, A., Lambright, D. G., Hamm, H. E. \& Sigler, P. B. Crystal structure of a G-protein beta gamma dimer at 2.1A resolution. Nature 379, 369-374 (1996).

17. Hamm, H. E. \& Gilchrist, A. Heterotrimeric G proteins. Curr. Opin. Cell Biol. 8, 189-196 (1996).

18. Sanada, K. \& Tsai, L. H. G protein betagamma subunits and AGS3 control spindle orientation and asymmetric cell fate of cerebral cortical progenitors. Cell 122, 119-131 (2005).

19. Sachdev, P. et al. G protein beta gamma subunit interaction with the dynein light-chain component Tctex-1 regulates neurite outgrowth. EMBO J. 26, 2621-2632 (2007)

20. Schwindinger, W. F. \& Robishaw, J. D. Heterotrimeric G-protein betagammadimers in growth and differentiation. Oncogene 20, 1653-1660 (2001).

21. Mundy, D. I. \& Warren, G. Mitosis and inhibition of intracellular transport stimulate palmitoylation of a 62-kD protein. J. Cell Biol. 116, 135-146 (1992).

22. Nakamura, N. et al. Characterization of a cis-Golgi matrix protein, GM130. J. Cell Biol. 131, 1715-1726 (1995).

23. Lyssand, J. S. \& Bajjalieh, S. M. The heterotrimeric [corrected] G protein subunit $\mathrm{G}$ alpha $\mathrm{i}$ is present on mitochondria. FEBS Lett. 581, 5765-5768 (2007).

24. Andreeva, A. V., Kutuzov, M. A. \& Voyno-Yasenetskaya, T. A. G alpha12 is targeted to the mitochondria and affects mitochondrial morphology and motility. FASEB J. 22, 2821-2831 (2008).
25. Chen, Q., Turner, J., Watson, A. J. \& Dive, C. v-Abl protein tyrosine kinase (PTK) mediated suppression of apoptosis is associated with the up-regulation of Bcl-XL. Oncogene 15, 2249-2254 (1997).

26. Zheng, C. et al. MAPK-activated protein kinase-2 (MK2)-mediated formation and phosphorylation-regulated dissociation of the signal complex consisting of p38, MK2, Akt, and Hsp27. J. Biol. Chem. 281, 37215-37226 (2006).

27. Chen, H. et al. Mitofusins Mfn 1 and Mfn2 coordinately regulate mitochondria fusion and are essential for embryonic development. J. Cell Biol. 160, 189-200 (2003).

28. Karbowski, M., Norris, K. L., Cleland, M. M., Jeong, S. Y. \& Youle, R. J. Role of Bax and Bak in mitochondrial morphogenesis. Nature 443, 658-662 (2006).

29. Karbowski, M. et al. Quantitation of mitochondrial dynamics by photolabeling of individual organelles shows that mitochondrial fusion is blocked during the Bax activation phase of apoptosis. J. Cell Biol. 164, 493-499 (2004).

30. Setoguchi, K., Otera, H. \& Mihara, K. Cytosolic factor- and TOM-independent import of C-tail-anchored mitochondrial outer membrane proteins. EMBO J. 25, 5635-5647 (2006).

31. Dell, E. J. et al. The betagamma subunit of heterotrimeric $\mathrm{G}$ proteins interact with RACK1 and two other WD repeat proteins. J. Biol. Chem. 277, 4988849895 (2002).

32. Tieu, Q. \& Nunnari, J. Mdvlp is a WD repeat protein that interacts with the dynamin-related GTPase, Dnmlp, to trigger mitochondrial division. J. Cell Biol. 151, 353-366 (2000).

33. Roychowdhury, S. \& Rasenick, M. M. G protein betalgamma2 subunits promote microtubule assembly. J. Biol. Chem. 272, 31576-31581 (1997).

34. Bozidis, P., Williamson, C. D. \& Colberg-Poley, A. M. Isolation of endoplasmic reticulum, mitochondria, and mitochondria-associated membrane fractions from transfected cells and from human cytomegalovirus-infected primary fibroblasts. Curr. Protoc. Cell Biol. Chapter 3, Unit 327 (2007).

\section{Acknowledgments}

We thank Drs Aimin Zhou from Cleveland State University, Shencai Lin from Xiamen University, Heping Cheng from Peking University and Junjie Hu from Nankai University for their constructive suggestions and critical reading of the manuscript. We wish to thank Professor Xun Shen for his advice on the FRET assay. We also thank all laboratory members for their discussions and comments. We would like to appreciate Dr Margerate Fuller (Stanford University) for the Mfn plasmids, and Dr David Chan (California Institute of Technology) for Mfn knockout cell lines. The Chen Laboratory is supported by a key project from the Nature Science Foundation of China (no. 30630038, 90713006) and the National Proprietary Basic Research Program (973 program project, Grant nos. 2007CB914800, 2011CB910903).

\section{Author contributions}

J.Z. performed all the experiments. W.L. helped with the immunoprecipitation assay, and J.L. helped with constructs. W.X helped with electron microscopy experiment, and X.S assisted with mitochondrial fractionation assay. L.L and C.J helped in data discussion. Q.C. oversaw the project, designed the experiments and wrote the manuscript.

\section{Additional information}

Supplementary Information accompanies this paper on http://www.nature.com/ naturecommunication

Competing financial interests: The authors declare no competing financial interests

Reprints and permission information is available online at http://npg.nature.com/ reprintsandpermissions/

How to cite this article: Zhang, J. et al. G-protein $\beta 2$ subunit interacts with mitofusin 1 to regulate mitochondrial fusion. Nat. Commun. 1:101 doi: 10.1038/ncomms1099 (2010) 